\title{
Medios de comunicación y medio ambiente en México*
}

\author{
Francisco Javier Martínez Garza** \\ Recibido: 2019-02-07 • Enviado a pares: 2019-02-20 \\ Aprobado por pares: 2019-03-14 - Aceptado: 2019-05-20 \\ https://doi.org/10.22395/angr.v18n35a4
}

\begin{abstract}
Resumen
Al considerar que los medios de comunicación contribuyen para que los ciudadanos participen con actitud positiva en la mejora de las condiciones medioambientales, el presente trabajo se planteó como objetivo principal identificar las características que distinguen la información relacionada con el medio ambiente, tal y como se difundió en México, en cinco medios de comunicación durante 2015, 2016 y 2017. El estudio, realizado con la técnica del análisis de contenido cuantitativo, incluyó las noticias y reportajes relacionados con el medio ambiente presentados en los periódicos Excélsior y El Norte, y en los telediarios nocturnos de TV Azteca, Televisa y Canal 11. La muestra incluyó el análisis de una semana construida de forma aleatoria durante cada uno de los años. Los resultados refieren que en México los medios de comunicación, sobre todo las televisoras, se han olvidado de comunicar a la sociedad los temas orientados a la participación en la conservación del medio ambiente. Sin embargo, sí suelen prestar atención especial al tema cuando se trata de un evento al cual, por sus características, se le puede añadir cierta dosis de espectacularidad (incendios, inundaciones, muertes, etc.). Los contenidos medioambientales en los medios mexicanos, además de limitados, carecen de diversidad, tanto en los temas como en los actores o las fuentes a las que recurren. A lo anterior habría que añadir el poco compromiso que manifiestan para promover, entre sus lectores o su público, una cultura social orientada a conservar y preservar el medio ambiente.
\end{abstract}

Palabras clave: cambio climático; medios de información; gestión ambiental; prensa de información diaria; noticiero de televisión; análisis de contenido.

* Este artículo se desprende de un proyecto de investigación realizado a finales del 2017, el cual se planteó como objetivo principal identificar la representación del cambio climático en la prensa y noticieros de televisión de España y México. El trabajo lo patrocinó la Universidad de Castilla la Mancha, en Cuenca, España y por el Tecnológico de Monterrey, México. El autor agradece la participación en la investigación de Elizabeth Tiscareño García, Lilia Paola del Real Villarreal y Luís G. Frías Gámez, estudiantes del Doctorado en Estudios Humanísticos del Tecnológico de Monterrey.

** Doctor en Periodismo y Comunicación Social, Universidad de Sevilla, España. Profesor investigador del Tecnológico de Monterrey, México. Miembro del Sistema Nacional de Investigadores, nivel 1, Monterrey, México. Correo electrónico: francisco@itesm.mx. Orcid: https://orcid.org/0000-0001 $7405-9724$ 


\title{
Mass Media and Environment in Mexico
}

\begin{abstract}
Considering that mass media contribute to the participation of citizens in improving environmental conditions with a positive attitude, this work was developed to identify the features that distinguish information related to the environment, as it was broadcasted in Mexico, in five mass media during 2015, 2016 and 2017. The study, conducted with the quantitative content analysis technique, included news and reports related to the environment that were presented in the newspapers Excelsior and El Norte, and on the nightly news broadcasts of TV Azteca, Televisa, and Canal 11. The sample included the analysis of a randomly constructed week for every one of those years. The results refer to the fact that in Mexico, the mass media (especially the television media) have forgotten to participate in society with topics oriented to the conservation of the environment. However, they do tend to pay special attention to the subject in case of events that, due to its characteristics, can add a certain dose of spectacularity (fires, floods, deaths, etc.). In addition to being limited, the environmental content in the Mexican mass media lack diversity, both in the themes and actors or the sources they turn to. In addition to this, we should add the little commitment they show to promote among their readers or their public, a social culture oriented to conserve and preserve the environment.
\end{abstract}

Keywords: climate change; mass media; environmental management; daily press information; tv news; content analysis.

\section{Meios de comunicação e meio ambiente no México}

\begin{abstract}
Resumo
Ao considerar que os meios de comunicação contribuem para que os cidadãos participem com atitude positiva na melhoria das condições ambientais, o presente trabalho tem como objetivo principal identificar as características que distinguem a informação relacionada com o meio ambiente, exatamente como foi difundida no México em cinco meios de comunicação durante os anos 2015, 2016 e 2017. O estudo, realizado com a técnica de análise de conteúdo quantitativo, incluiu as notícias e as reportagens relacionadas ao meio ambiente que foram apresentadas nos jornais Excélsior e El Norte, além dos telejornais noturnos da Tv Azteca, Televida e Canal 11. A amostra incluiu a análise de uma semana construída de forma aleatória para cada um dos anos. Os resultados apontam que os meios de comunicação no México, sobretudo as televisões, esqueceram-se de apresentar à sociedade os temas orientados à participação na conservação do meio ambiente. No entanto, costumam dar atenção especial ao tema quando se trata de um evento ao qual, por suas características, é possível adicionar certa dose de espetacularidade (incêndios, inundações, mortes etc.). Os conteúdos ambientais nos meios mexicanos, além de limitados, carecem de diversidade, tanto nos temas quantos nos agentes ou nas fontes a que recorrem. A isso, deveríamos adicionar o pouco compromisso que manifestam para promover, entre seus leitores ou seu público, uma cultural social orientada a conservar e preservar o meio ambiente.
\end{abstract}

Palavras-chave: câmbio climático; meios de informação; gestão ambiental; imprensa de informação diária; notícias de televisão; análise de conteúdo. 


\section{Introducción}

El incremento global de la temperatura se reconoce desde hace poco más de tres décadas como uno de los principales problemas que enfrenta la humanidad. Su impacto se refleja cada vez más frecuentemente en eventos como sequías, huracanes de alto riesgo, incendios forestales, etc., con lo cual contribuye a la generación de problemas vinculados con la pobreza, el desarrollo económico y la salud. Para contrarrestar los efectos, desde la Organización de Naciones Unidas (ONU) se ha promovido una serie de programas, entre los cuales destaca la Convención Marco de las Naciones Unidas sobre el Cambio Climático - cmnucc (ONU, 1992). De ella se desprendieron dos de los más importantes acuerdos mundiales: el Protocolo de Kyoto y los Acuerdos de París (ONU, 2014), este último adoptado por 195 países (ONU, 2018).

Las evidencias sugieren que la actividad humana es la principal causante del calentamiento global (Encinas, 2011). De ahí que la Organización de las Naciones Unidas para la Educación, la Ciencia y la Cultura (Unesco) solicitó a los países firmantes promover programas orientados a educar, sensibilizar y concientizar a las personas sobre el tema (edn - Unesco, 2006). Con la intención de promover entre los ciudadanos una actitud favorable hacia el medio ambiente, la Unesco decretó, entre 2005 y 2014 , el "Decenio para la Educación del Desarrollo Sostenible". Entre las sugerencias que planteó la Unesco para alcanzar mejores resultados destaca la de involucrar a los ministerios de educación, la sociedad civil organizada, las ong, empresas privadas y medios de comunicación. Se presupone que cuanto más conocimiento tengan las personas sobre el tema, más fácilmente podrían adoptar las políticas públicas o los programas que requieran de su participación. México fue uno de los primeros países en adherirse a los acuerdos del CMNUCC, de manera que una vez concluida la vigencia del programa en cuestión, se podría esperar que los mexicanos mantuvieran una visión diferente a la que tenían sobre el tema. Sin embargo, estudios demoscópicos recientes realizados en el país refieren que, aun cuando a los ciudadanos les preocupa el medio ambiente, no lo comprenden del todo (Zamora, 2018).

Entre los mexicanos prevalece un desinterés sobre el tema del medio ambiente. Esta situación se podría atribuir al desconocimiento que se tiene sobre el mismo, ya que de contar con más información, se podría esperar que estos modificaran la actitud, valores y comportamiento que tienen respecto al tema. Dados los aspectos señalados, en este trabajo se propone conocer las características que distinguen a la información que se difundió sobre el medio ambiente en distintos medios de comunicación mexicanos durante el periodo 2015-2017.

La realización de un estudio enfocado en los mensajes vinculados con el medio ambiente obedece a la necesidad que existe en México por mejorar la situación que prevalece en la materia. Conocer las características que le distinguen permitirá tomar 
acciones benéficas para la situación, y mejorar con ello la información que reciben los mexicanos sobre este tema en particular. Lo anterior toma relevancia, sobre todo, si se considera que México es uno de los países más afectados por el cambio climático, por lo que existe la necesidad de tomar acciones más contundentes sobre ello. Los informes señalan que 319 de los municipios del país están en situación de "muy alta" o "alta" vulnerabilidad (Inecc, 2013). De acuerdo con el mismo informe, 13 \% del total de los municipios padecen serios eventos climáticos (inundaciones, deslaves, sequías y ondas de calor), situaciones que en poco tiempo afectarán severamente la productividad agrícola y la disponibilidad de agua para consumo humano.

Las condiciones que prevalecen en México ponen de manifiesto que se ha subestimado el problema relacionado con el medio ambiente. En la actualidad, la situación exige que este sea considerado un problema de prioridad nacional, de manera que se precisa cuidar las áreas nacionales protegidas, erradicar la deforestación, establecer normas de calidad del aire en las ciudades, generar políticas públicas para crear y ejecutar planes ambiciosos de reciclaje que estimulen una drástica reducción en la generación de basura (Carabias, citado en Hernández Mares, 2018). Son muchos los problemas vinculados con el medio ambiente que exigen la atención del gobierno, sociedad e instituciones, para evitar que se continúe con el deterioro. Sin embargo, pareciera que este no termina por ser reconocido entre la población; esto incluye a los líderes de opinión, quienes tampoco le reconocen del todo. Lo anterior se refleja en la agenda de trabajo que presentaron los contendientes a la presidencia de la república durante la campaña electoral federal del 2018, en la que medio ambiente y sustentabilidad fueron dos de los tópicos menos abordados (Sánchez, 2018).

El estado mexicano tiene la mayor obligación de poner el tema en cuestión en la discusión pública — conforme los ciudadanos lo perciban como un asunto de mayor envergadura, seriedad y preocupación- y que se le incorpore en la agenda de instituciones y actores políticos, seguramente así comenzará a ocupar un mayor espacio en la agenda periodística (González Alcaraz, 2012). Las condiciones del país en la materia exigen que se promueva un consenso entre la población, aspecto en el que los medios podrían contribuir notablemente, ya que estos se mantienen aún como el principal referente informativo de la mayoría de mexicanos (Ifetel, 2017; Díez Nicolás, 2004).

Desde la propuesta de la agenda setting se presupone que, en tanto los medios promocionen el tema en sus contenidos, el público conocerá del mismo y podría desarrollar una actitud más positiva en torno a la situación medioambiental. De acuerdo con la misma propuesta teórica, la importancia que le concedan los medios a un tema podría contribuir para que su público le tenga más presente (McCombs y Shaw, 1972). De manera que, si se busca que los ciudadanos estén más enterados 
sobre el particular, se precisa que las instituciones de medios incluyan en su agenda informativa mensajes de este tipo. Por el contrario, de no hacerlo, el tema al cual se alude, difícilmente podría incorporarse en la arena pública (González, A., 2012).

La ausencia de contenidos que promuevan el conocimiento del medio ambiente parece ser una constante en los medios de comunicación, ya que estos suelen limitar su inclusión en sus contenidos; y aún más, cuando lo hacen suelen privilegiar mensajes que contribuyen a incrementar el tamaño de su audiencia (Piñuel, 2013; Lozano y Carabaza, 2009; León, 2007). La política editorial que han asumido los medios termina por favorecer eventos cuyas características permiten dotarlos de cierta dosis de sensacionalismo. De ahí que cuando se aluda al medio ambiente en los medios, se los relacione principalmente con grandes catástrofes de flora y fauna, sequías, incendios forestales, huracanes, olas de calor, inundaciones, etc. Se trata, en todos los casos, de mensajes que poco contribuyen a la formación de la población sobre la verdadera naturaleza del cambio climático (León, 2007; Francescutti, et al., 2013). Bajo ese esquema, en realidad, "los medios han sido poco eficaces atendiendo la falta de información" (Francescutti et al., 2013, p. 28).

Sobre este último aspecto, la propuesta de la agenda setting sostiene que la imagen que el público se forma de un tema no depende exclusivamente de la cantidad, el tiempo o la frecuencia con la que se presenta, sino de la manera como se presenta la información al público. En lo que se ha denominado el 2. nivel de la agenda setting se considera que el encuadre periodístico, es decir, las características o los atributos que los medios confieren al mensaje, repercute en la percepción que el público llega a tener del mismo (Lozano, 2007). En esta última perspectiva se presupone que el tratamiento periodístico de un tema afecta la comprensión que las personas adquieran de él (Aruguete, 2017).

Desde la propuesta de la teoría de la agenda setting, se considera por lo tanto que, la prominencia del objeto, esto es, la incorporación del tema en el contenido de los medios, y la transferencia de los atributos que envuelven al evento, tal y como se presenta, impacta en la imagen que el público llega a tener sobre el evento (Mccombs, López y Llamas, 2003, p. 59). Por lo anterior es importante considerar los atributos que se destacan en los mensajes relacionados con el medio ambiente, ya que estos contribuirán a conformar las imágenes que el público se haga del mismo (McCombs y Shaw, 1972).

Las evidencias indican que la mayoría de los mensajes que se difunden sobre el medio ambiente en los medios de comunicación se caracterizan por estar ungidos de aspectos sensacionalistas; de otra forma este tipo de contenidos difícilmente sería incluido en su agenda, por cuanto carece de atracción para el público (González Cruz, 2007). Las evidencias indican también que, además de limitar la presencia de men- 
sajes relacionados con el medio ambiente, la información que se presenta, se suele concentrar más en el suceso que en el proceso; difícilmente existe algún seguimiento periodístico sobre los eventos, y sus contenidos suelen ser aislados, fragmentados y descontextualizados (Rodríguez-Cruz y Bezunartea Valencia, 2015; Díaz Nosty, 2009; Carabaza, 2007; González A., 2012; González C., 2007). Así entonces, la manera en la que se conducen los medios limita o distorsiona la presencia de los contenidos que reclaman la necesidad de proteger y contribuir a la mejora del medio ambiente, como lo sugiere el principio 19 de la CMNUCC (González A., 2012).

Para responder al cuestionamiento planteado en el marco de esta investigación se integraron las siguientes preguntas, cada una de las cuales tenía como propósito identificar las características que distinguen la información medioambiental, tal como se la difunde en prensa y en los telediarios mexicanos:

a) ¿En qué medida destacan los medios de comunicación en México la información relacionada con el medio ambiente?

b) ¿Qué tan variada es la oferta de contenidos medioambientales que se difunden en medios de comunicación del país?

c) ¿Qué características distinguen a la información relacionada con el medio ambiente que se transmite al público mexicano?

En términos generales, las preguntas señaladas contribuirían a identificar, en primer lugar, la densidad de la información que existe sobre el tema en los medios de comunicación de México; en segundo lugar, a conocer la diversidad de los temas medioambientales que presentan en las mismas instituciones; y en tercer lugar, a describir cómo se presenta la información relacionada con el tema en los espacios informativos del país. Es importante destacar que la muestra representa el análisis de un número limitado de medios de comunicación, de manera que los alcances del estudio se deberán tomar como una referencia de la situación, mas no como una representación del accionar de los medios mexicanos en su totalidad.

\section{Metodología}

Para realizar el estudio se recurrió a la técnica del análisis de contenido cuantitativo (Kerlinger, 1986; Wimmer y Dominick, 1996), ya que permite comparar sistemáticamente los mensajes que se difundieron en prensa y en noticieros de televisión. Para efectos del trabajo, se tomaron como unidad de análisis las noticias o reportajes en los cuales se aludió al tema del medio ambiente. La muestra incluyó la información de prensa y noticieros de televisión. En el caso de la primera se incluyeron dos periódicos mexicanos, uno regional y otro de la ciudad de México; y con respecto a los segundos, tres noticieros nocturnos de la televisión nacional mexicana se incluyeron 
en la muestra, todos ellos de la capital del país. En la selección de los periódicos se consideró, además de la circulación, que contaran con una versión digital similar a la que presentaba el diario impreso. Lo anterior permitiría recuperar los ejemplares que circularon años atrás.

Con lo anterior, la muestra de periódicos quedó constituida por los periódicos El Norte de Grupo Reforma, publicado en la Ciudad de Monterrey y Nuevo León, y Excélsior, de la Ciudad de México. En cuanto a los telediarios, se consideró que además de la penetración contaran con una plataforma digital, situación que permitiría videograbar los contenidos de años anteriores; entonces, la muestra se compuso de los informativos nocturnos de Televisa (Canal 2), TV Azteca (Canal 13) y Canal 11, este último de la televisión pública.

Como tamaño de la muestra, el trabajo construyó aleatoriamente una semana para cada uno de los años 2015, 2016 y 2017. Se eligió trabajar con el muestreo de semana aleatoria compuesta (Lozano, 1994) a fin de evitar sesgos derivados de la aparición de un hecho periodístico extraordinario. Con base en el modelo señalado, se construyó una semana aleatoria de los años 2015 y 2016, y otra del primer semestre del 2017. Las fechas se utilizaron por igual en los cinco medios. Debido al procedimiento señalado, la muestra finalmente se constituyó con 45 emisiones de telediarios nocturnos y de 30 periódicos matutinos. Para la codificación se contó con la participación de tres estudiantes del Doctorado en Estudios Humanístico del Tecnológico de Monterrey, cada uno de los cuales recibió una preparación previa, y posteriormente se realizó una prueba de fiabilidad, cuyo resultado ascendió a 87 \% (Holsti, 1969).

\section{Resultados}

\section{El medio ambiente en medios de México}

Durante los tres años abarcados en el análisis, en los diarios y en los noticieros de televisión mexicanos se difundieron 105 mensajes relacionados con la temática estudiada, 58 \% de los cuales fueron publicados por la prensa. En términos generales, esto es, considerada la totalidad de los mensajes, cada uno de los medios incluidos en el estudio difundió siete notas por semana en promedio. Sin embargo, es importante destacar que existe una notable diferencia en la proporción de tiempo y espacio que destinó cada una de las instituciones a la difusión del tema.

El periódico Excélsior transmitió la mayor cantidad de mensajes relacionados con el medio ambiente durante el periodo de la muestra. Su participación aportó la tercera parte del total de los contenidos que finalmente comprenden el tamaño de la muestra. La cantidad de información que presentó Excélsior a sus lectores es ligeramente inferior a la que ofrecieron el total de televisoras juntas (tabla 1). 
Tabla 1. Proporción de la información relacionada con el medio ambiente y su ubicación en los medios mexicanos

\begin{tabular}{|c|c|c|c|c|c|c|}
\hline & Medio & N. publicaciones & N. ${ }^{\circ} \%$ & $\mathrm{~N} .^{\circ}$ de párrafos & $\%$ portada & $\%$ interiores \\
\hline \multirow{4}{*}{ Prensa } & El Norte & 24 & 39 & 200 & 4 & 96 \\
\hline & Excélsior & 37 & 61 & 269 & 16 & 84 \\
\hline & Total & 61 & 100 & 469 & $\mathrm{~N} / \mathrm{A}$ & N/A \\
\hline & Medio & $\mathrm{N} .^{\circ}$ de menciones & N. ${ }^{\circ} \%$ & $\mathrm{~N} .^{\circ}$ de minutos & $\% 1$.er segmento & $\begin{array}{c}\% \text { otros seg- } \\
\text { mentos }\end{array}$ \\
\hline \multirow{4}{*}{ Telediarios } & Canal 2 & 9 & 20 & 14 & 22 & 78 \\
\hline & Canal 11 & 18 & 41 & 32 & 41 & 59 \\
\hline & Canal 13 & 17 & 39 & 28 & 44 & 56 \\
\hline & Total & 44 & 100 & 74 & $\mathrm{~N} / \mathrm{A}$ & N/A \\
\hline
\end{tabular}

Fuente: elaboración propia.

La cantidad de información que se presenta sobre el medio ambiente sugiere que tanto los telediarios como la prensa mexicana dispensan poca atención a los contenidos de este tipo. Igualmente, la mayor parte de los mensajes se estructuran tomando como referencia el formato de la noticia ( $93 \%$ ), de manera que es raro que los medios recurran a géneros como la entrevista (1\%) o el reportaje (2\%), cuyas características favorecen la participación y, por ende, podrían aportar mayor cantidad de información.

Además de compartir poca información sobre el medio ambiente, los medios suelen ubicarla en espacios considerados como "poco relevantes", esto es, desde el punto de vista periodístico. Lo anterior se desprende si se repara que, en caso de la prensa, nueve de cada diez mensajes relacionados con el tema en cuestión se publicaron en páginas interiores. La situación no es distinta en los telediarios: dos de cada tres notas se dieron a conocer a partir del tercer segmento (tabla 1).

A lo anterior correspondería añadir el espacio tan limitado que se brinda al tema en cuestión, sobre todo si se considera que durante los periodos analizados los periódicos publicaron un total de 22.504 párrafos', 469 de los cuales (2 \%) aludían a información relacionada con el medio ambiente. Se trata de información que en la mayoría de los casos se publicó en páginas interiores, ya que las pocas notas que aparecieron en

Esta cifra no incluye la información publicada dentro de las secciones relacionadas con el deporte, editoriales, espectáculos, cultura, seguridad pública ni avisos de ocasión. 
portada se referían a eventos matizados de sensacionalismo: inundaciones, desastres y eventualidades atmosféricas, etc.

En lo que corresponde a la televisión, sus noticieros participaron con una política editorial muy similar a la que adoptó la prensa. De 2.835 minutos que dispensaron los informativos a transmisión de información durante el período de análisis, poco menos del $3 \%$ se orientaron a transmitir información sobre el medio ambiente.

Las evidencias indican también que cuando los medios de comunicación informan sobre el medio ambiente en México, suelen privilegiar contenidos relacionados con siniestros como incendios forestales, inundaciones, huracanes, etc. Al adoptar esta política editorial, finalmente las instituciones relegan otros eventos o acontecimientos desde los cuales se podría promover una cultura más favorable hacia el tema. Por ejemplo, participar en la inclusión de contenidos relacionados con congresos, ciclos de conferencias, entrevistas a expertos, difusión de la ciencia, etc.

Otro aspecto que también caracteriza a la información que se transmite en los medios de comunicación mexicanos analizados sobre el medio ambiente radica en que la mayoría de ellos (97 \%) alude a eventos o acontecimientos que tienen lugar en el país. Esta tendencia se suele mantener inamovible; solo se rompe cuando un acontecimiento ocurrido fuera de la república mexicana permite brindarle un tratamiento periodístico que atraiga la atención del público — por ejemplo, la presencia de un "super huracán" o la "sequía que acaba con la vida de miles de personas en otros sitios del mundo"一.

\section{La agenda del medio ambiente en los medios mexicanos}

Vistos el tiempo y el espacio destinados por los medios de comunicación a la transmisión de información relacionada con el medio ambiente durante el período de análisis, es posible señalar que no existe una clara política editorial, diseñada ex profeso, para incluir con mayor frecuencia contenidos relacionados con este tópico, ya que estos suelen ajustar su agenda de acuerdo a la manera como se presentan los acontecimientos. Luego, si en un periodo surgen muchas eventualidades, la cantidad de información no incrementa; por el contrario, al no existir, decrece.

La mayor parte de los mensajes que se presentaron en los medios analizados se dieron a conocer durante el 2015. Al siguiente año, la cantidad de esta disminuyó considerablemente, e incrementó de nuevo durante 2017, pero sin superar la cantidad registrada durante el primer período. Esta misma tendencia se repite en los telediarios, en los que tampoco se refleja un interés por promover la información que contribuya a la conformación de una cultura medioambiental entre los mexicanos (tabla 2). 
Tabla 2. Relación de tiempo y espacio que dedicaron durante los diferentes períodos al medio ambiente en los medios de comunicación en México

\begin{tabular}{ccccc}
\hline \multirow{2}{*}{ Año de estudio } & \multicolumn{3}{c}{ Prensa } & \multicolumn{3}{c}{ Telediarios } \\
\cline { 2 - 5 } & Notas & Párrafos & Notas & Tiempo \\
\hline 2015 & 25 & 178 & 14 & 1.279 \\
\hline 2016 & 16 & 133 & 9 & 1.395 \\
\hline 2017 & 20 & 158 & 21 & 1.734 \\
\hline Total & 61 & 469 & 44 & 4.408 \\
\hline
\end{tabular}

Fuente: elaboración propia.

La ausencia de una política editorial orientada a promover mensajes relacionados con el medio ambiente en los medios de comunicación del país, se refleja también en la poca diversidad que caracteriza a este tipo de contenidos. Pareciera que los medios de comunicación limitan la transmisión de este tipo de mensajes a los fenómenos meteorológicos (inundaciones, ciclones, huracanes, sequías, etc.) o en su defecto, a destacar los estragos de la contaminación. Poco más de la mitad de los mensajes (54 \%) se relacionan con las categorías anteriores (tabla 3). Otro tercio de la información está sustentada en contenidos en los cuales se alude a la flora y la fauna y, en menor medida, la problemática relacionada con el agua (34\%). Mientras que la del calentamiento global, es la categoría menos cubierta, su participación representó el $1 \%$ del total de los temas que se abordaron, tanto en la prensa como en los telediarios.

La contaminación y los servicios de meteorología son los dos tópicos vinculados al medio ambiente que más frecuentemente aparecen en los medios del país. Estos mismos son también los que suelen aparecer con más frecuencia en las portadas de los periódicos o en el primer segmento de los telediarios. Más de la mitad de la información relacionada con estos dos temas se presentó durante el primer segmento de los telediarios, y poco menos de dos terceras partes se ubicaron en la portada de prensa.

Las evidencias indican también, que existe una diferencia muy notable entre los contenidos que se difunden en la prensa y los que aparecen en los noticieros de televisión. Mientras que en la prensa se suele destacar el tema de la contaminación y el problema del agua, en los telediarios se priorizan los contenidos relacionados con los fenómenos meteorológicos (tormentas, inundaciones y ciclones). En lo que sí coinciden es que, en ambos casos, los mensajes se suelen revestir de ciertas dosis de sensacionalismo para atraer la atención del público lector y televidente.

La prensa y los telediarios coinciden en los criterios editoriales mediante los cuales evalúan el valor de la noticia: en ambos casos se suelen privilegiar los hechos 
o acontecimientos que por sus características representan una atracción para su público, sin profundizar en aspectos técnicos como las consecuencias o el impacto que acarrea este tipo de fenómenos en la salud o en la economía, solo por citar algunos.

Tabla 3. Tiempo y espacio que concedieron los medios

a los temas relacionados con el medio ambiente

\begin{tabular}{|c|c|c|c|c|c|}
\hline \multirow{2}{*}{ Tema } & \multirow{2}{*}{$\begin{array}{c}\% \text { total } \\
\text { de mensajes }\end{array}$} & \multicolumn{2}{|c|}{ Prensa } & \multicolumn{2}{|c|}{ Telediarios } \\
\hline & & $\%$ de mensajes & \% de párrafos & $\%$ de mensajes & $\%$ de tiempo \\
\hline Contaminación & 27 & 31 & 32 & 21 & 29 \\
\hline Flora y fauna & 18 & 20 & 18 & 16 & 18 \\
\hline Calentamiento global & 1 & 2 & 2 & 0 & 0 \\
\hline Agua & 15 & 23 & 23 & 5 & 4 \\
\hline Meteorología & 29 & 16 & 14 & 46 & 34 \\
\hline Otros & 11 & 8 & 11 & 14 & 15 \\
\hline Total & $\begin{array}{c}100 \\
(\mathrm{n}=105)\end{array}$ & $\begin{array}{c}100 \\
(\mathrm{n}=61)\end{array}$ & $\begin{array}{c}100 \\
(n=469)\end{array}$ & $\begin{array}{c}100 \\
(n=44)\end{array}$ & $\begin{array}{c}100 \\
(\mathrm{n}=74 \text { min. })\end{array}$ \\
\hline
\end{tabular}

Fuente: elaboración propia.

\section{El mensaje sobre el medio ambiente}

Los medios de comunicación no suelen incluir mensajes en los que se aluda a los principales tratados y acuerdos internacionales relacionados con la prevención del medio ambiente. Durante el periodo estudiado solo apareció publicado un mensaje, en el cual se aludió a los Acuerdos de París. En la misma nota se presentaron algunas referencias en las que se aludía a las consecuencias que acarrearía el detrimento del medio ambiente. Sin embargo, en ninguna parte del mensaje se aludió a la amenaza o riesgo que representa para los ciudadanos esta situación. También son pocos los contenidos en los que se presentaron sugerencias o alternativas para mejorar las condiciones del medio ambiente; $y$ tampoco se criticaron o se aludió a las actividades humanas que las lesionan.

Los medios de comunicación suelen recurrir a las fuentes periodísticas para sustentar la información que difunden a sus lectores o televidentes. El análisis realizado en torno a los contenidos de la prensa y los noticieros de televisión, indica, que casi tres de cada cuatro mensajes incluyeron, como mínimo, la opinión de alguna fuente periodística. Sin embargo, la mitad de ellas corresponden a personas ligadas con alguna institución gubernamental, o a participantes del evento al que se atiende (tabla 4). Esto es, no recurrieron a fuentes especializadas, las cuales seguramente podrían ofrecer una mejor explicación sobre el particular. 
Tabla 4. Proporción, espacio y tiempo que se les conceden a las diferentes fuentes cuando se alude al medio ambiente en los medios de comunicación

\begin{tabular}{lccccc}
\hline & Fuentes & \multicolumn{2}{c}{ Prensa } & \multicolumn{2}{c}{ Telediarios } \\
\hline \multicolumn{1}{c}{ Fuentes de la muestra } & $\begin{array}{c}{ }^{\circ} \% \\
\text { publicaciones }\end{array}$ & \% de párrafos & \% de fuentes & \% de minutos & \% de fuentes \\
\hline Académicos & 16 & 17 & 15 & 17 & 18 \\
\hline Grupo político & 29 & 30 & 32 & 24 & 23 \\
\hline Sociedad civil organizada & 9 & 14 & 11 & 4 & 5 \\
\hline Testigos y ciudadanos & 11 & 9 & 8 & 14 & 18 \\
\hline Servicios de asistencia & 24 & 25 & 26 & 20 & 18 \\
\hline Experto & 11 & 6 & 8 & 22 & 18 \\
\hline Total & 100 & 100 & 100 & 100 & 100 \\
\hline & $(\mathrm{n}=75)$ & $(\mathrm{n}=420)$ & $(\mathrm{n}=53)$ & $(\mathrm{n}=54)$ & $(\mathrm{n}=22)$ \\
\hline
\end{tabular}

Fuente: elaboración propia.

Durante el periodo de análisis, ningún académico fue consultado por los periodistas para atender temas como las energías renovables o sustentables, las implicaciones de la contaminación en la salud y el manejo de las fuentes hidráulicas o de los manglares, entre otros. En todos los casos, y como se expresó en el párrafo anterior, cuando se presentó la ocasión, los medios recurrieron a fuentes gubernamentales o a personal que laboraba en el sitio donde ocurrió el evento. Aún más, los resultados muestran que cuando se trata del medio ambiente, ni académicos, ni miembros de la sociedad civil organizada - y esto incluye a personas ligadas a algunas ONG — tienen oportunidad de participar como fuentes periodísticas. La manera como se conducen las empresas informativas, termina por ofrecer información del medio ambiente muy sesgada, con lo que se afecta la calidad de la información que se transmite a los ciudadanos al presentar una visión limitada sobre el problema del medio ambiente.

\section{Conclusión}

México es uno de los países con mayor deterioro medioambiental; de ahí la importancia de promover la inclusión de este tipo de mensajes, los cuales podrían favorecer el conocimiento que los ciudadanos tienen sobre el tema. Lo anterior es importante porque se presupone que cuanto más conozcan sobre el particular, más posibilidades tendrían de adoptar una mejor actitud para poner en práctica las políticas públicas que se instrumenten.

La Unesco considera a los medios de comunicación uno de los principales instrumentos a los que pueden recurrir los gobiernos para concientizar a la sociedad sobre las repercusiones que podría acarrear el deterioro ambiental. Sin embargo, al 
menos en el caso de México, estas instituciones han contribuido de manera limitada a participar de esta función. A tres años de concluido el programa Decenio para la educación del desarrollo sostenible, promovido por la Unesco, los mexicanos conocen poco del tema; y aunque parecen convencidos de la importancia que representa participar en favor de este, son pocos quienes están convencidos y decididos a sacrificar su comodidad.

La Unesco recomendó a los países participantes del cmNUCC que promovieran, entre otras acciones, la participación de los medios de comunicación, en tanto que estos podrían contribuir a transmitir al público la importancia que representa el medio ambiente en su calidad de vida — desde la propuesta de la agenda setting — (McCombs y Shaw, 1972). Se precisa que estas instituciones incorporen el tema en su agenda informativa, situación que, de acuerdo con las evidencias académicas, no ha ocurrido (Piñuel, 2013; Lozano y Carabaza, 2009; León, 2007). Los resultados de algunos estudios indican también que, además de limitada, la información que se ofrece sobre el tema, es aislada y descontextualizada (Rodríguez-Cruz, 2012; Díaz Nosty, 2009; Carabaza, 2007; González A., 2012; González C., 2007).

El análisis realizado a los mensajes que transmitieron los tres informativos nocturnos de la televisión y los dos diarios mexicanos durante el periodo estudiado coincide con los aspectos y argumentos anteriores: se hace evidente que en México los medios limitan la presencia de contenidos relacionados con el medio ambiente, y cuando lo hacen suelen privilegiar temas que les permitan añadir altas dosis de sensacionalismo. Estos resultados coinciden con los obtenidos por otros autores ubicados más allá de la frontera de México. Es decir, se reafirma una vez más el desinterés de los medios de comunicación por incluir el tema en su agenda informativa.

Vista la cantidad de mensajes que se presentan sobre el medio ambiente tanto en la prensa como en los noticieros de televisión, el espacio y el tiempo que se destinaron a tales menesteres apenas sobrepasa el 3 \% del total de la información que difundió cada uno de ellos durante el periodo analizado. Por lo anterior, es posible señalar que las instituciones de medios concedieron poco interés al tema ya que, además de ser limitados, la mayoría de los contenidos se publicó en las páginas interiores en los periódicos o en los segmentos secundarios en los noticieros de televisión.

Desde la perspectiva de la propuesta de la agenda setting, lo anterior puede contribuir para que los ciudadanos consideren el medio ambiente como un tema de poco interés, o que incluso lleguen a menospreciarlo (Aruguete, 2017; Lozano, 2007). Wolf (1991) sostiene que los mismos periodistas o las instituciones de medios asignan el sitio en el que se presentará la información, así como el tiempo o el espacio que se le brindará. Tomado como referencia el valor de la noticia al que alude Wolf, el resultado del estudio permite deducir que los medios mexicanos no han reparado en la importancia que representa su papel en la transmisión de la información medioambiental, en cuanto a la percepción que el público se forme sobre el tema. 
El estudio encuentra también que, además de limitar la cantidad de información y ponerla en opciones poco relevantes, los mensajes se suelen relacionar con temas o acontecimientos a los que se podría revestir de una buena dosis de sensacionalismo, situación con la que coinciden autores que han estudiado este aspecto (Piñuel, 2013; Lozano y Carabaza, 2009; León, 2007). Las evidencias muestran que cuando se trata el tema, se le suele relacionar con información referida a huracanes, inundaciones, sequías, desaparición de especies de la flora y la fauna, etc. Es decir, se refuerza la idea que prevalece: las instituciones de medios buscan promover contenidos que favorezcan su comercialización, en lugar de ofrecer información didáctica sobre el tema.

La información difundida sobre el medio ambiente en los medios de comunicación del país mantiene una tendencia periodística rutinaria; la mayoría de los mensajes se apoyan en las fuentes participantes al evento. Es común, por lo tanto, que cuando se aluda al tema de la contaminación o al problema del agua, los periodistas acudan con quienes tradicionalmente fungen como sus fuentes periodísticas, esto es, gobernantes, políticos o empleados del gobierno. Mediante este proceder, los medios descartan la oportunidad de atender los puntos de vista de los expertos o los profesionales del tema, los cuales podrían contribuir a formar una opinión más aterrizada sobre el particular. El hecho de privilegiar la presencia de fuentes como las señaladas impacta directamente en el conocimiento del público de este país, ya que se limita a conocer información tratada desde el punto de vista político, sin reparar en otra perspectiva, que podrían brindar fuentes alternativas a las tradicionalmente empleadas.

La limitada exposición que se ofrece sobre el tema, aunada a la poca variedad con la que se le representa, finalmente se han convertido en elementos que limitan que el público acceda a puntos de vista desde los cuales se promueva el tema, a la vez que contribuyan a colocarlo en sus agendas. Esta situación no es exclusiva de los medios mexicanos, ya que coincide con un accionar común entre los medios (León, 2007; Francescutti et.al., 2013).

Más allá de resaltar las catástrofes ambientales o problemas meteorológicos, si se tratara de calificar el desempeño que han tenido las instituciones de medios en lo que corresponde al medio ambiente, es posible señalar que su aporte didáctico ha sido pobre y ofrecen a su público una perspectiva condicionada sobre el problema. Por lo anterior, es posible señalar que los mexicanos no han tenido la oportunidad de acceder a mensajes en los que se presenten propuestas o alternativas sobre el deterioro del medio ambiente en aras de concientizarlos.

Más allá de que México haya sido uno de los primeros países firmantes de la CMNUCC y que, por tanto, haya sido instado a implementar el programa Decenio para la educación del desarrollo sostenible, pareciera que el Estado mexicano no adoptó una acción para promover entre los medios la necesidad de potenciar este tipo de mensajes; esta es, por lo tanto, una tarea pendiente. 


\section{Referencias}

Aruguete, N. (2017). Agenda Building. Revisión de la literatura sobre el proceso de construcción de la agenda mediática. Signo y Pensamiento, 36(70), 36-52.

Carabaza, J. (2007). Apuntes para comprender la cultura ambiental desde la comunicación. Global Media Journal, 3(6), 1 1 10.

Carabias, J. (2018). Los desafíos ambientales de México para el 2018 [entrevista]. En Hernández Mares, P. Mongabay Latam. Periodismo ambiental independiente. Recuperado de: https://es.mongabay. com/2018/01/los-desafios-ambientales-mexico-2018/

Díaz Nosty, B. (2009). Cambio climático, consenso científico y construcción mediática. Los paradigmas de la comunicación para la sostenibilidad. Revista Latina de Comunicación Social, 64, 99-119.

Díez Nicolás, J. (2004). El dilema de la supervivencia. Los españoles ante el medio ambiente. Caja de Madrid.

Ecoosfera. México (2018). Elecciones México. Medio Ambiente, Sustentabilidad y Ecología. Ecoosfera. México. Recuperado de: https://ecoosfera.com/2018/04/mexico-candidatos-presidentesecologia-medioambiente-sustentabilidad/

ED Unesco (2006). Decenio de la Naciones Unidas de la educación para el desarrollo sostenible (2005-2014). Plan de Aplicación Internacional. Recuperado de: http://unesdoc.unesco.org/ images/0014/001486/148654so.pdf

Encinas Malagón, M. D. (2011). Medio ambiente y contaminación. Principios básicos. UPV. Recuperado de: https://addi.ehu.es/bitstream/handle/10810/16784/Medio\%20Ambiente\%20y\%20 Contaminaci\%C3\%B3n.\%20Principios\%20b\%C3\%Al sicos.pdf?sequence=6.

Francescutti, L. P., Tucho Fernández, F., e Iñigo Jurado, A. I. (2013). El medio ambiente en la televisión española: análisis de un año de informativos. Estudios sobre el mensaje periodístico, 19(2), 683-701. https://doi.org/10.5209/rev_ESMP.2013.v19.n2.43492

González Alcaraz, L. (2012). Medio ambiente y agenda mediática. Oportunidades y barreras para la cobertura periodística de cuestiones ambientales en la prensa local. Oficios Terrestres: revista electrónica sobre ciencias sociales desde la comunicación y la cultura, 1(28). Recuperado de: http://perio. unlp.edu.ar/ojs/index.php/oficiosterrestres/article/view/1612

González Cruz, E. (2007). Los medios de comunicación y la ecología en México. El Cotidiano, 146, 43-51. Recuperado de: http://www.elcotidianoenlinea.com.mx/pdf/14606.pdf

Hernández Mares, P. (2018, enero). Los desafíos ambientales de México para el 2018 lentrevista con Caravias, J.] Mongabay Latam. Periodismo ambiental independiente. Recuperado de: https:// es.mongabay.com/2018/01/los-desafios-ambientales-mexico-2018/

Holsti, O. R. (1969). Content Analysis for the Social Sciences and Humanities. Addison-Wesley.

Instituto Federal de Telecomunicaciones (IFT) (2017). Encuesta Nacional de Consumo de Contenidos Audiovisuales 2016. IFT . Recuperado de: http://www.ift.org.mx/sites/default/files/encca2016_vfcompressed.pdf.

Instituto Nacional de Ecología y Cambio Climático (Inecc) (2013). Desarrollo de la Estrategia Nacional de Adaptación al Cambio Climático. Gestión de Riesgos ante el Cambio Climático y Diagnóstico de 
Vulnerabilidad. México: Inecc, Qbic, Global Green Growth Institute. Recuperado de: https://www. gob.mx/cms/uploads/attachment/file/4 1978/Estrategia-Nacional-Cambio-Climatico-2013.pdf

Kerlinger, F. N. (1986). Foundations of behavioral research ( $3^{\circ}$ ed.). Holt, Rinehart y Winston.

León, B. (2007). El Medio Ambiente en las televisiones españolas. Un análisis de contenido de los informativos nacionales. En F. R. Contreras, Cultura Verde. Ecología, Cultura y Comunicación (pp. 361-373). Consejería del Medio Ambiente.

Lozano, J. C. (1994). Hacia la reconsideración del análisis de contenido en la investigación de los mensajes comunicacionales. En C. Cervantes y E. Sánchez Ruiz (coords.), Investigar la comunicación: propuestas iberoamericanas (pp. 135 158). Universidad de Guadalajara.

Lozano, J. C. (2007). Teoría e investigación de la Comunicación de masas (2 ed.). Pearson Prentice Hall.

Lozano, J. C., y Carabaza, J. (2009). La televisión y su impacto en la cultura ambiental en audiencias de Monterrey, Guadalajara y Cd. De México. En Comunicación y Medio Ambiente. Reflexiones, análisis y propuestas (pp. 46-97). Tecnológico de Monterrey.

McCombs, M., López-Escobar, E., y Llamas, J. P. (2003). Establecimiento de la agenda de atributos. En M. McCombs e I. Luna Pla (eds.), Agenda Setting de los medios de comunicación (pp. 57-82). Universidad de Occidente.

McCombs, M., y Shaw, D. (1972). The Agenda-Setting Function of Mass Media. Public Opinion Quarterly, 36, 176-187.

Organización de Naciones Unidas (ONU) (1992). Convención marco de las Naciones Unidas sobre el cambio climático. ONU. Recuperado de: https://unfccc.int/resource/docs/convkp/convsp.pdf

Organización de Naciones Unidas (ONU) (2014). La ONU y el cambio climático. Recuperado de: http:// www.un.org/climatechange/es

Organización de Naciones Unidas (ONU) (2018). La ONU y el cambio climático. Hacia un acuerdo sobre el clima. Recuperado de: http://www.un.org/climatechange/es/hacia-un-acuerdo-sobreel-clima/index.html

Piñuel Raigada, J. L. (2013). El discurso hegemónico de los Media sobre el "Cambio Climático" (Riesgo, Incertidumbre y Conflicto) y estrategias de intervención. En R. Mancinas Chávez y R. Fernández Reyes (coords), Medios de comunicación y cambio climático. Actas de las Jornadas Internacionales (pp. 27-44). Facultad de Comunicación de la Universidad de Sevilla. Recuperado de: https://idus.us.es/xmlui/handle/1 1441/36858

Rodríguez Cruz, I., y Bezunartea Valencia, M. J. (2015). El medio ambiente, supeditado a la conveniencia de la economía y la política. Diagnóstico de la información a partir de sus fuentes. Revista Zer, 20(39), 85-100. Recuperado de: http://www.ehu.eus/ojs/index.php/Zer/ article/view/15523/14500

Sánchez, J. A. (2018, mayo 30). Candidatos ignoran a medio ambiente, acusan activistas. Milenio. Recuperado de: https://www.milenio.com/elecciones-mexico-2018/candidatos-ignoran-medioambiente-acusan-activistas

Wimmer, R. D., y Dominick, J. R. (1996). La investigación científica de los medios de comunicación: Una introducción a sus métodos. Bosch Comunicación. 
Wolf, M. (1991). Tendencias actuales del estudio de medios. Diálogos de la comunicación, (30), 26-30.

Zamora Sáenz, I. (2018). Valoraciones sociales sobre el cambio climático en México. Visor Ciudadano, 57. Instituto Belisario Domingo, Saneado de la República. Recuperado de http://bibliodigitalibd. senado.gob.mx/bitstream/handle/123456789/3888/NC_57.pdf?sequence = 1 EisAllowed =y 